\title{
Stereoselective Synthesis of All-Carbon Tetrasubstituted Alkenes
}

\section{Key words}

lithium

magnesium

palladium

tetrasubstituted alkenes

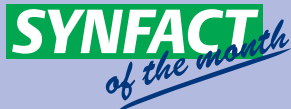<smiles>[R]C([R])C(=O)Oc1c(C)cccc1C</smiles>

$$
\begin{aligned}
& \text { BuLi (1.1 equiv) } \\
& \mathrm{R}^{3} \mathrm{Li} \text { (1.2 equiv) } \\
& -78 \text { to }-20^{\circ} \mathrm{C} \\
& \overrightarrow{\mathrm{Tf}_{2} \mathrm{O} \text { or } \mathrm{CIP}(\mathrm{O})(\mathrm{OR})_{2}} \\
& \text { (2.5 equiv) } \\
& -20 \text { to } 22^{\circ} \mathrm{C}
\end{aligned}
$$<smiles>[R]C([R])=C([2H])[2H]</smiles>

up to $95 \%$ yield ZIE up to $>20: 1$

$$
\begin{gathered}
\mathrm{R}^{4} \mathrm{M}(2.5 \text { equiv) } \\
{[\mathrm{Pd}](2 \text { or } 10 \mathrm{~mol} \%)} \\
\mathrm{X}(4 \text { or } 10 \mathrm{~mol} \%) \\
\hline \text { solvent, } 22^{\circ} \mathrm{C}, 40^{\circ} \mathrm{C} \text { or } 70^{\circ} \mathrm{C}
\end{gathered}
$$<smiles>[R]C=C([R])[2H]</smiles>

up to $90 \%$ yield Z/E up to $>20: 1$

$\mathrm{G}=\mathrm{Tf}$ or $\mathrm{P}(\mathrm{O})(\mathrm{OR})_{2}(\mathrm{R}=\mathrm{Et}, \mathrm{Ph})$

$\mathrm{M}=\mathrm{MgBr}\left[\mathrm{G}=\mathrm{P}(\mathrm{O})(\mathrm{OR})_{2}\right] ; 9-\mathrm{BBN}, \mathrm{B}(\mathrm{OH})_{2}, \mathrm{H}(\mathrm{G}=\mathrm{Tf})$

$[P d]=P d(d b a)_{2}\left[G=P(O)(O R)_{2}\right] ; \operatorname{Pd}(O A c)_{2}, P{ }^{2} C_{2}(P P h)_{3}(G=T f)$

$X=X P h o s\left[G=P(O)(O R)_{2}\right]$; SPhos, Cul $(G=T f)$

$\mathrm{R}^{1}=\mathrm{Ph}, 4-\mathrm{MeOC}_{6} \mathrm{H}_{4}, 2-\mathrm{MeC}_{6} \mathrm{H}_{4}$, 3-thienyl, $i-\mathrm{Pr}, t-\mathrm{Bu}$

$\mathrm{R}^{2}=\mathrm{Et}, \mathrm{Me}, \mathrm{Bn}, i-\mathrm{Pr}$

$\mathrm{R}^{3}=n-\mathrm{Bu}, \mathrm{Ph}, \mathrm{C}(\mathrm{Me})=\mathrm{CH}_{2}$, 2-furanyl

$\mathrm{R}^{4}=\mathrm{Ph}, \mathrm{Tol}, \mathrm{Me}, \mathrm{Bn}, n$-Hex, ethynylphenyl

Selected examples:<smiles>CCC(O)=C(CC)c1ccccc1</smiles>

$66 \%$ yield ZIE > 20:1<smiles>CCC(=C(c1ccccc1)c1ccc(C)cc1)c1ccccc1</smiles>

$52 \%$ yield

$$
Z / E=19: 1
$$

$\mathrm{G}=\mathrm{P}(\mathrm{O})(\mathrm{OR})_{2}$<smiles>CCC(OCCOc1ccccc1)=C(OCCO)c1ccccc1</smiles>

$56 \%$ yield Z/E > 20:1<smiles>COc1ccc(/C(C)=C(\Cc2ccccc2)c2ccccc2)cc1</smiles>

$$
52 \% \text { yield }
$$$$
Z / E=19: 1
$$
$\mathrm{G}=\mathrm{P}(\mathrm{O})(\mathrm{OR})_{2}$<smiles>CCOC(OCC)=C(C)c1ccccc1C</smiles>

$84 \%$ yield ZIE $>20: 1$

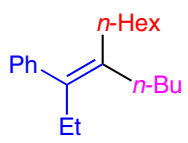

$89 \%$ yield ZIE $>20: 1$ $\mathrm{G}=\mathrm{Tf}$<smiles>CCCCCCCCOC(OCCCC)=C(C)C(C)(C)C</smiles>

\section{$48 \%$ yield} Z/E $>20: 1$<smiles>CCC(C#Cc1ccccc1)=C(CC)c1ccccc1</smiles>

$90 \%$ yield $Z I E>20: 1$ $\mathrm{G}=\mathrm{Tf}$
Significance: The stereoselective synthesis of various all-carbon tetrasubstituted alkenes is disclosed, employing a two-step protocol which includes the stereoselective generation of an alkenyl pseudohalide followed by stereospecific palladium-catalyzed cross-coupling. The appropriate tetrasubstituted alkenes are obtained in good yield and with good diastereomeric ratios, providing mainly the Z-isomers.
Comment: The reaction is proposed to proceed via a ketene intermediate which is stereospecifically attacked by a lithium organyl to form the trisubstituted enolate moiety attached to a pseudohalide group [OTf or $\left.\mathrm{OP}(\mathrm{P})(\mathrm{OR})_{2}\right]$. This moiety may be converted into other functional groups by palladium-catalyzed cross-couplings to give the corresponding all-carbon tetrasubstituted alkenes.

SYNFACTS Contributors: Paul Knochel, Nadja M. Barl 